\title{
HCFA: Hybrid Coupled Flat Architecture for QoS Enhancement of Multimedia Applications in Mobile WiMAX Broadband Access Network
}

\author{
K. Chandrakala \\ Research scholar, Dept of ECE \\ Pondicherry Engineering College \\ Pondicherry, India
}

\author{
D. Saraswady, $\mathrm{PhD}$ \\ Professor, Dept. of ECE \\ Pondicherry Engineering College \\ Pondicherry, India.
}

\begin{abstract}
Worldwide Interoperability for Microwave Access (WiMAX) is the fourth generation (4G) core technology based on the IEEE 802.16 family. The challenge in the coming years for mobile networks will be to offer high bitrates data services to customers in mobility. Future mobile architectures are being standardized to offer mobility between heterogeneous access technologies. The design of these architectures does not take into account the scalability requirement since they are centralized with many network levels and dependency. Existing and future generations of wireless technologies are competition for providing seamless computing solutions using mobility management. To perform fast and seamless handovers for efficient data transfer there is a need for good mobility and session management framework. The high latency and high cost are the major drawback of a centralized high speed $3 \mathrm{G} / 4 \mathrm{G}$ network. On the other hand, flat architecture of $3 \mathrm{G} / 4 \mathrm{G}$ does not support high speed mobility even though it provides less latency. This paper proposes a novel architectural design using the entity, namely adaptive convergence router (ACR) for both low latency and seamless mobility which is introduced in the existing WiMAX flat architecture. It stores information and simultaneously checks for resource availability and handoff control for every second and maintains the database, and adaptively controls the session handoff with better QoS. The mobile station sends resource request message to the ACR and it grants recourses according to the available bandwidth. So according to the proposed novel architecture, users can share three simultaneous paths according to their needs. The real time users can use centralized path, non real time users can share flat architecture path and combined hybrid path can be used for multimedia users. This mechanism provides load sharing and reduces the congestion so that less latency with seamless mobility can be achieved. The performance of the proposed architecture is evaluated through simulation by using OPNET modeler considering the metrics like throughput and delay
\end{abstract}

\section{Keywords}

MWiMAX, mobility, scalability, flat architecture, QoS

\section{INTRODUCTION}

WiMAX is originating from the IEEE 802.16 standard which specifies wireless interface MAN with its range of spectrum 10-66GHZ with physical layer and medium control layer. Later this standard structure is named as WiMAX (Wireless Interoperability for Microwave Access).[1] Wireless broadband access with high speed, low cost, and more reliability can be achieved through this standard and it can also extendable through our normal fiber optic communication system. This standard eliminates the line of sight problem that exists in the frequency range of $2 \mathrm{GHz}-$ $11 \mathrm{GHz}$ with licensed and unlicensed frequencies [2][3]. This standard is further extended to mobile WiMAX and formed as IEEE 802.16e. The mobile WiMAX provides the facility for effective mobility management by which it allows the terminal to roam in to different service locations for call delivery without loss of connection and facilitates mobility management. Mobility management consists of two methodologies: one is location management and other is handoff management. Paging and registration is the two important functions of location management. Registration is the process of identifying the location of the user equipments (UE) for incoming and outgoing calls handling of paging (or) arranging the UE at the time of call setup. Modifying the systems to follow the mobile terminal attachment points between the successive communications is the key function of location management.

\section{WiMAX TECHNOLOGY}

The IEEE 802.16 standard [4] has emerged as an important technology for delivering packet data service in a wide area cellular network. IEEE 802.16 was initially targeted for fixed wireless deployments, but the 802.16e-2005 amendment published in early 2006 introduced significant enhancements to enable the support of mobility for the OFDMA physical layer. In order to promote interoperability among products based on the IEEE 802.16 standard and manage certification of vendor equipments, the WiMAX forum was formed. The forum has the important role of defining interoperability tests for a subset of the 802.16 features, and making arrangements for third party certification labs to test vendor equipments. The forum also defines standard interfaces for upper protocol layers, as the scope of the IEEE 802.16 standard only encompasses the MAC and PHY layers. WiMAX uses OFDM-MIMO, [5] which is also emerging as the main technology for future cellular packet data networks, including 3GPP Long Term Evolution (LTE) and 3GPP2 RevC Air Interface Evolution (AIE). The introduction of OFDM MIMO in WiMAX carries with it a number of considerations for handoff management that may be different from those in existing 3G CDMA systems. Those factors are briefly addressed here. Resource control in OFDM-MIMO networks is done at the lowest level in the network hierarchy, at the base station, with frame-by-frame scheduling. The implications to handoff frame-by-frame scheduling on both reverse link and forward link mainly concern soft handoff, where multiple APs are simultaneously either transmitting or receiving from a given mobile. If soft handoff is supported, tight coordination between the APs in soft handoff is required if the benefits of dynamic scheduling are to be maintained. On the forward link, the soft handoff transmissions may be either 
in the same resource block measured in OFDM tone-symbol in multiple APs, which allows signal combining at the radio frequency level (as long as the differential delay between sites is within the cyclic prefix duration), or in different resource blocks, in which case the mobile listens to both allocations and performs soft combining after detection. On the reverse link, the multiple APs must detect and combine the terminal's transmission, and care must be taken by the AP schedulers not to place two terminals in the same resource block allocation. This requires tight coordination between the APs. The introduction of MIMO to next-generation wireless systems for the most part does not influence the support of handoff, but it can allow for more flexibility. An example of this is the supports of MIMO on the forward link where the multiple transmit antennas are on different APs. From the terminal perspective, the signal is processed in the same way as if the two (or more) transmit antennas were on the same AP, but there is an extra degree of flexibility on the network side. Of course, this requires the tight coordination of transmissions from multiple APs.

\section{MWIMAX ARCHITECTURES}

The MWiMAX forum is working on the implementation of a MWiMAX mobility architecture supporting both homogeneous and heterogeneous mobility. The mobility and handover management framework depends on the choice of suitable network deployment architecture. A brief discussion on the different potential MWiMAX deployment architectures is presented here. In a MWiMAX centralized architecture where a subnet consists of one Access Network Gateway (ASN GW) and multiple BSs under its control. The ASN GW has centralized control of the subnet. The IP-layer functionalities are also located in the individual ASN GWs, which efficiently support seamless handover along with low latency micro and macro-mobility activities. In the flat architecture a subnet consists of exactly one BS and one ASN GW. The IP-layer functionalities are located in the individual BSs. The architecture supports macro-mobility and handover with optional session anchoring capabilities.

\subsection{Benefits of Flat Architecture}

- The controller - integrated BSs are connected directly to the core network (Internet), eliminating the requirements of the wireless technology dependent radio-access network (RAN). This is the access service network (ASN) in mobile WiMAX networks between the BS and the IP core network. The interoperability with heterogeneous wireless technologies is easy to achieve because the BSs are directly connected to the wireless technology-neutral IP core network.

- Flat architecture provides high scalability because there is new centralized performance bottleneck, while traffic is processed in a fully distributed fashion. It also provides adding or removing cells without concern about the capacity of the centralized controller to which the new BSs are attached. [6]

- Integrated design reduces the cost of intermediate communication. Also sophisticated cross-layer optimization is possible for performance gain.

- Flat architecture achieves resource efficiency gains by preventing suboptimal routing. Under the hierarchical architecture, all traffic must pass through the centralized controllers, which may extend the routing path, resulting in suboptimal traffic routing.
- Under flat architecture, single failure points (the centralized controller) do not exist, and the impact of a BS failure can be limited locally

- Flat architecture has economic advantages. The general purpose IP equipment is much cheaper than radio access network components because of economics of scale. The options for network management tools for IP networks are available at low cost as well.

\subsection{Challenges of flat architecture}

The challenges of flat architecture are handover performance, quality of service (QoS), self-configuration and selfoptimization and self diagnosis. Generally, IP mobility schemes are designed for macro mobility and produce relatively long handover delay, which is often too high for real-time applications like voice over IP (VoIP). A high performance IP-mobility solution for flat architectures needed. QoS provision is another challenge in the flat architecture. The IP network that connects BSs should be capable of proper QoS provision (bandwidth, delay) for satisfactory application control performance. In flat architecture, network configuration and resource management should be done in a distributed way, namely, selfconfiguration and self-optimization. This challenge comes with the benefits of scalability and flexibility. Related to self configuration and self-optimization, self-diagnosis is required for stable service provision in flat architecture networks. Failure diagnosis and recovery must be done in a distributed way, because BSs are not managed by centralized controllers.

\section{PROPOSED HYBRID COUPLED FLAT MWIMAX ARCHITECTURE}

Hybrid Coupled flat MWiMAX architecture shown in Figure 1 is a combination of both centralized and flat with adaptive convergence properties initialized in ACR. In a hybrid design, handoff control and radio resource control management is together performed by the ACR. It stores information and simultaneously checks for resource availability and handoff control, for every seconds and maintain the database, and adaptively controls the session handoff .It provides handshaking between the ACR and the MN. It sends authentication message for QoS control, controls the radio resource and the weak signal strength. It first checks for the received signal strength and throughput. If the BS loses the control, signal session handoff will be failed. This ACR overcomes the session failure by adapting control over the BS. ACR maintains all the registration details and subscriber information for the QoS provisioning. According to QoS information gathered ACR adapts to a network condition without handoff failure. When more number of users is entering the network, congestion occurs and session handoff fails sometimes. This mechanism provides load sharing and reduces the congestion so that less latency with seamless mobility can be achieved. Adding the IP function within the $\mathrm{BS}$ requires an optimized mobility mechanism as the $\mathrm{MN}$ will change its IP address each time it moves from one BS to another. The mobility support is also provided by the ACR and the received signal strength during call and the handover initiation takes place is also controlled. In this proposed model, ACR control the whole converging mechanism. The different base station has been searched by the mobile stations then the call is handover to the new base station without any failures. The new mobile station must be handover to the mobile station and then ACR also connects then gets the acknowledgement message. At last the data session will take place through the new base station with the help of ACR. 
When queuing and starving of resources occurs, ACR will adaptively initiate and session registration with privacy mechanism is performed. ACR stores information and simultaneously checks for resource availability and handoff control, for every seconds and maintain the database, and adaptively controls the session.

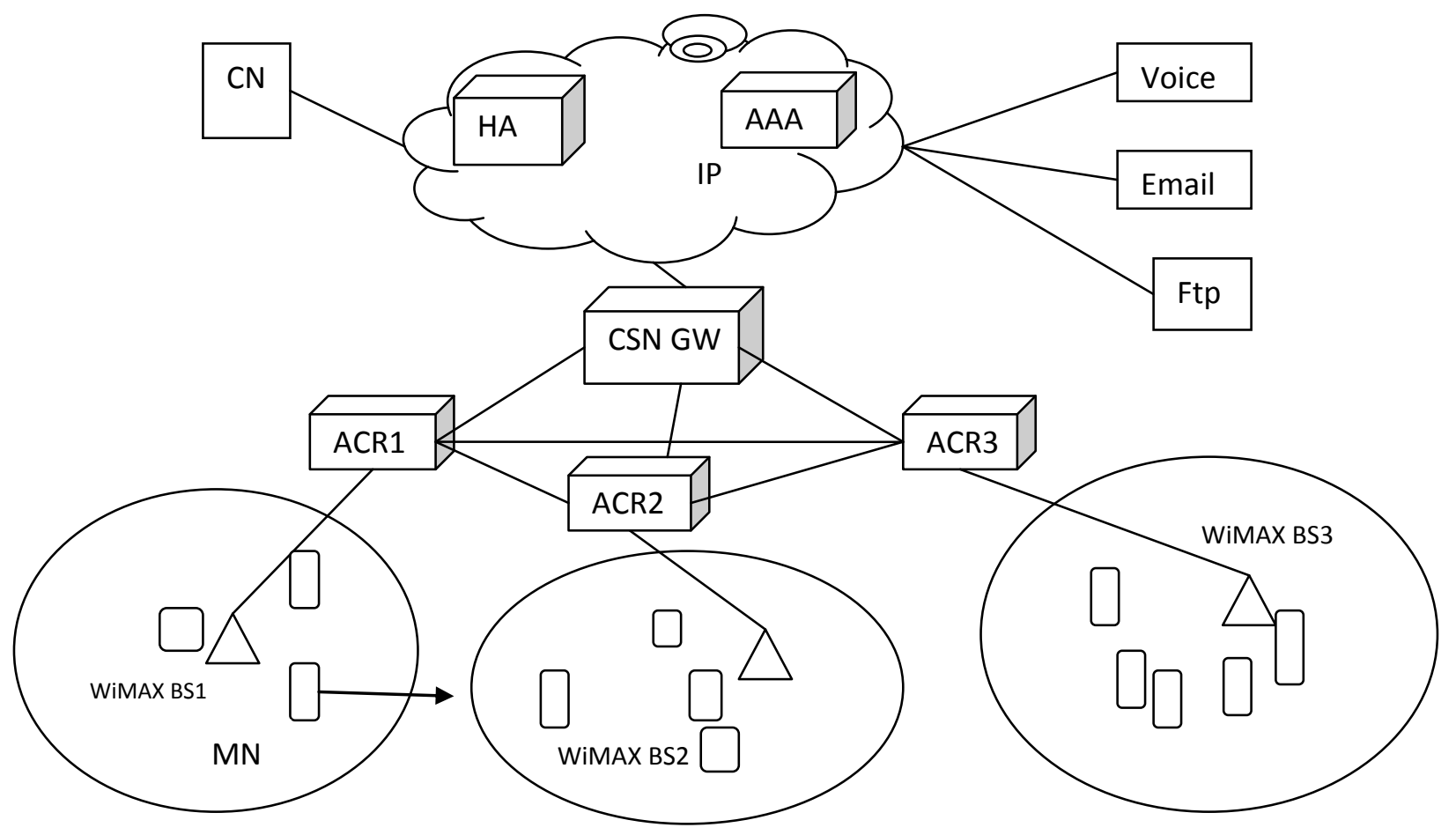

Fig 1: Hybrid coupled flat MWiMAX architecture

The Handoff flow for hybrid coupled flat architecture as shown in figure. 4

1. The MS is initially connected to an SBS. When the MS moves from its SBS to a TBS, the MS sends a HO_REQ (Handoff Request) message to the GW, indicating the need for an intra-GW handoff.

2. The SBS checks the resource availability with the TBS through RES_REQ (Resource Request) and RES_RSP (Resource Response) Messages. Once resources are confirmed, the GW initiates a SESS_XFER (Session Transfer) process.

3. Since the radio session is anchored at the GW, most of the session information is stored at the GW without the need for being transferred.

4. The GW then instructs the MS to execute the handoff through the HO_RSP (Handoff Response) message. The MS sends a HO_CMP (Handoff Complete) message to GW once the handoff is complete.

5. Once the link layer handoff is completed, the MS initiates a Mobile IP handoff to move the session anchoring point from the SAP/GW to the TAP. The data traffic will go through the new GW (TAP/GW) once both Layer 2 and Layer 3 handoffs are completed.

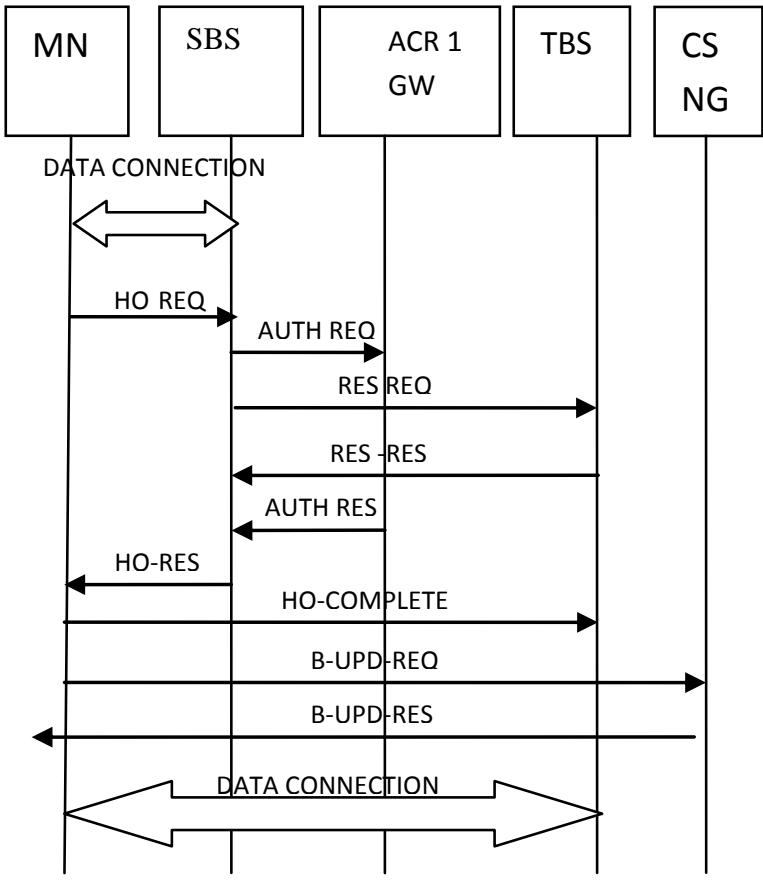

Fig 2: The Handoff flow for hybrid coupled flat MWiMAX architecture

\section{PERFORMANCE ANALYSIS}

To yield better results the proposed architecture is compared with existing networks. Table 1 shows the some of the list of simulation parameters used in the OPNET model. The performance metrics such as end-to-end delay, Jitter, Traffic 
received, throughput and packet loss ratio are considered for analyzing the proposed architecture.

Table 1: Simulation parameters

\begin{tabular}{|l|l|l|}
\hline $\begin{array}{c}\text { S. } \\
\text { No. }\end{array}$ & \multicolumn{1}{|c|}{ Parameters } & \multicolumn{1}{c|}{ values } \\
\hline 1. & Simulation area & $3000 \times 3000$ \\
\hline 2. & Operating frequency & $2500 \mathrm{MHz}$ \\
\hline 3. & Channel bandwidth & $10 \mathrm{MHz}$ \\
\hline 4. & No of base stations & 3 \\
\hline 5 & No of mobile stations & 20 \\
\hline 6 & Cell radius & $3000 \mathrm{~m}$ \\
\hline 7 & User mobility speed & $40 \mathrm{~km} / \mathrm{hr}$ \\
\hline 8 & Frame duration & $5 \mathrm{~ms}$ \\
\hline 9 & Traffic types & VoIP, email \\
\hline 10 & Modulation type & $64 \mathrm{QAM}$, OFDM \\
\hline 11 & Packet size & 512 \\
\hline
\end{tabular}

\subsection{Centralized Architecture}

In a centralized architecture, a subnet consists of one access network gateway and multiple base stations under its control. The ASN has centralized control of the subnet. The IP layer functionalities are also located in the individual ASN gate way. Since multiple nodes are involved in processing a call, call set up time is generally longer in a hierarchical network, ranging from several seconds to tens of seconds Here the delay is more since the base stations pass through a single gateway under a larger area single router cannot maintain the connectivity of all the base station as shown in Figure 3.

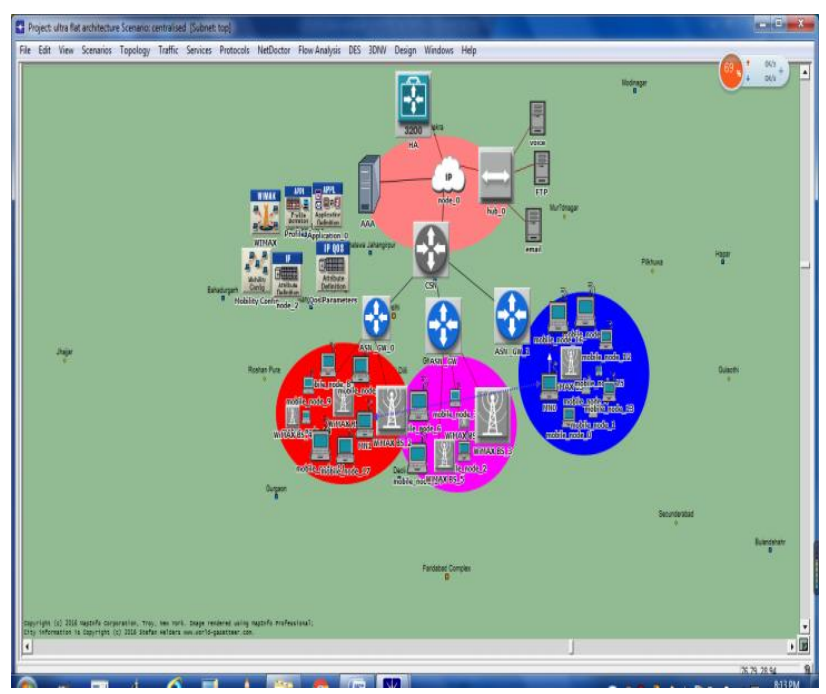

Fig 3: Centralized architecture

\subsection{Flat Architecture}

A subnet consists of exactly one base station and one ASN gate way. The IP functionalities are located in the individual BS. The AP and the GW which contains the RRC and the HO Control functions are integrated into one node .The network latency may be minimized as the number of nodes between the MS and the IP network is reduced to only one as shown in Figure. 4.

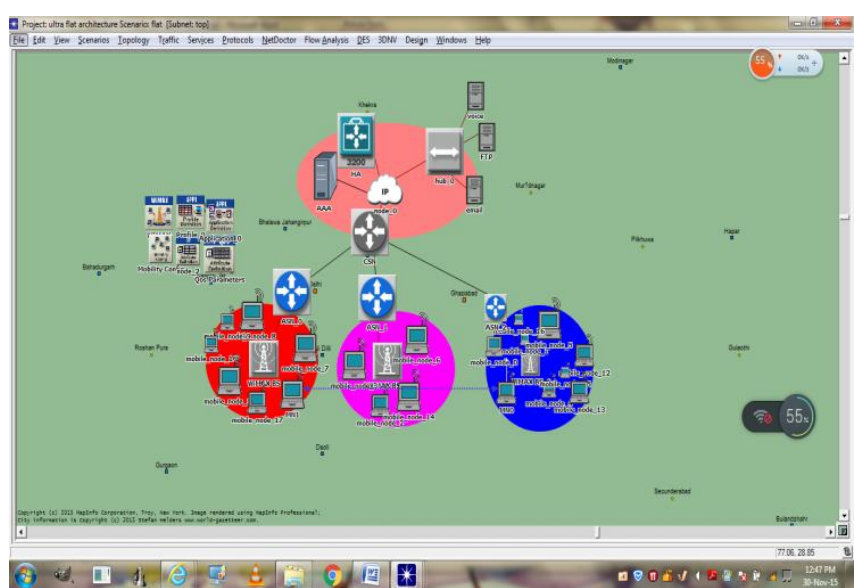

Fig 4: Flat architecture

\subsection{Hybrid Coupled Flat Architecture}

In order to reduce the end to end delay and packet drop adaptive convergence properties initialized in ACR. ACR control the whole converging mechanism for real time and non real-time services is proposed to enhance the QoS of WiMAX network. It stores information and simultaneously checks for resource availability and handoff control, for every seconds and maintain the database, and adaptively controls the session. When more number of users is entering the network, congestion occurs and session handoff fails. This mechanism provides load sharing and reduces the congestion so that low latency with seamless mobility can be achieved. The hybrid flat architecture is shown in Figure. 5

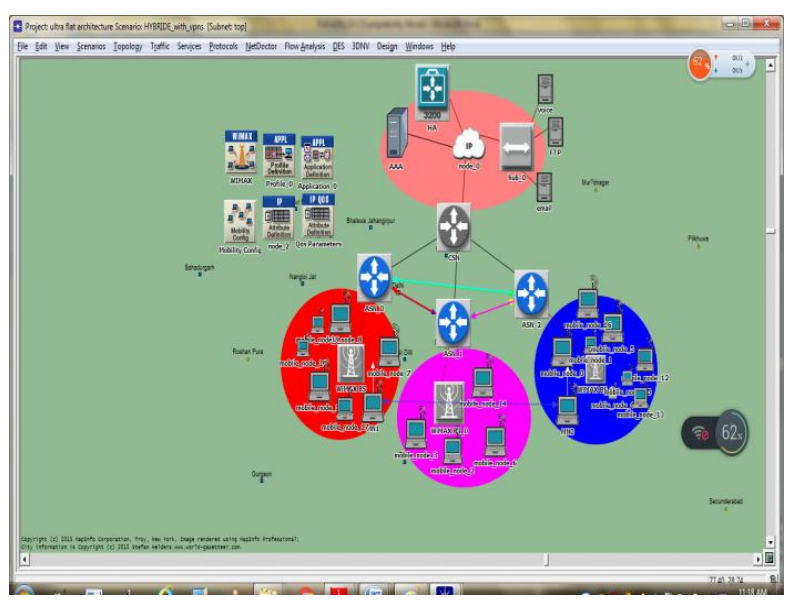

Fig 5: Hybrid coupled flat architecture

\subsection{End to end delay}

The end-to-end delay for existing and proposed architecture is simulated and comparison as shown in Fig. 6, it can be analyzed that Packet in voice is more in handoff as compared to a mobile network without handoff. Whenever a handoff occurs, it involves some basic steps like scanning, ranging, network re-entry and registration. There is no data transfer during scanning and transmission is further delayed due to ranging, network re-entry and registration process. So, all these factors sum up to increase the end to-end delay during handover. The end-to end delay for existing flat and centralized architecture is more when compared to proposed hybrid architecture. The delay is very less mainly 0.070 . The proposed architecture outperforms very well in terms of delay. 


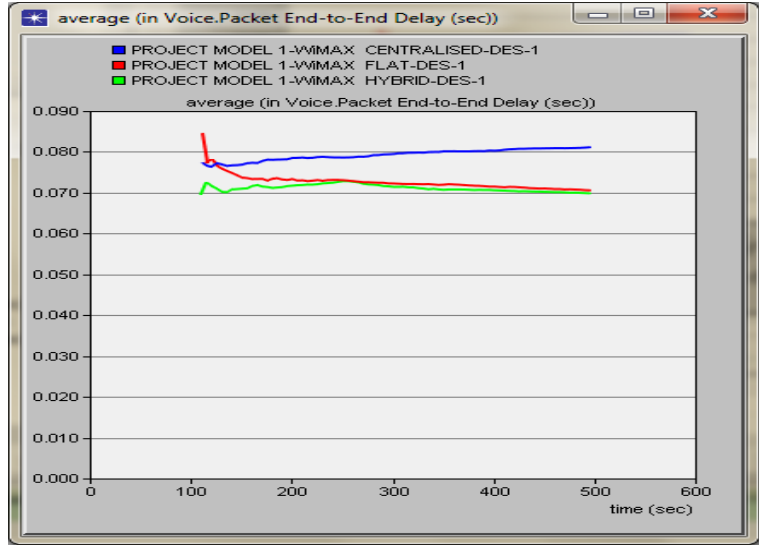

Fig 6: End- to-End delay for voice

\subsection{Jitter}

Jitter could be defined as the variation in delay or the variation in the time between packets arriving. The value of jitter is calculated from the end to end delay. Measuring jitter is an important way to determine the reliability of a network and the QoS the network offers. Jitter is normally used as an indicator of consistency and stability of a network. The jitter for existing flat and centralized architecture is more when compared to proposed hybrid architecture. Figure 7 shows the voice jitter comparison for the proposed and existing.

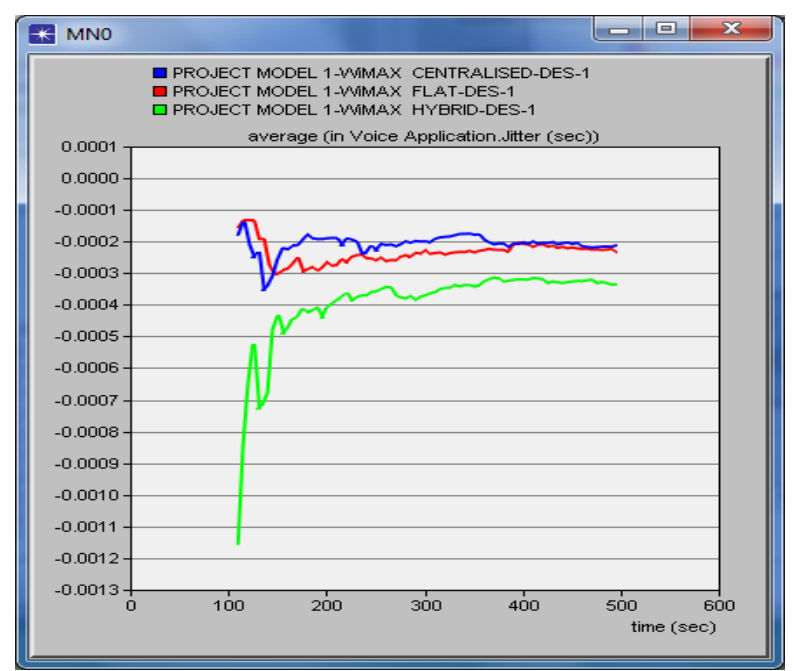

Fig 7: Voice Jitter

\subsection{Traffic received}

Traffic received is defined as the total number of packets successfully delivered to the destination the traffic received for existing flat and centralized architecture is less when compared to proposed architecture and it is shown in figure.9

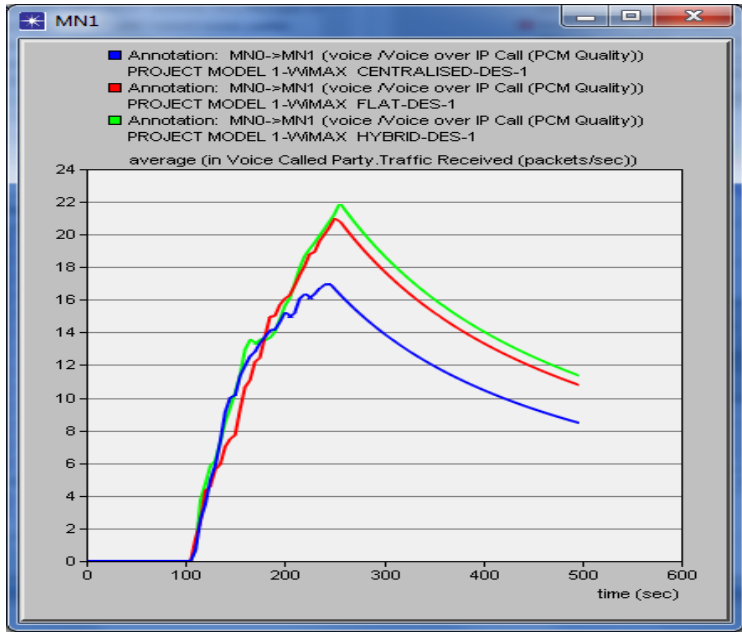

Fig 8: Voice traffic received

\subsection{Throughput}

Throughput is a measure of the number of packets successfully delivered in a network. It is measured in terms of packets/second. The value of throughput should be high for data transmission. The throughput for existing flat and centralized architecture is less when compared to proposed HCFA architecture and it is shown in figure 9

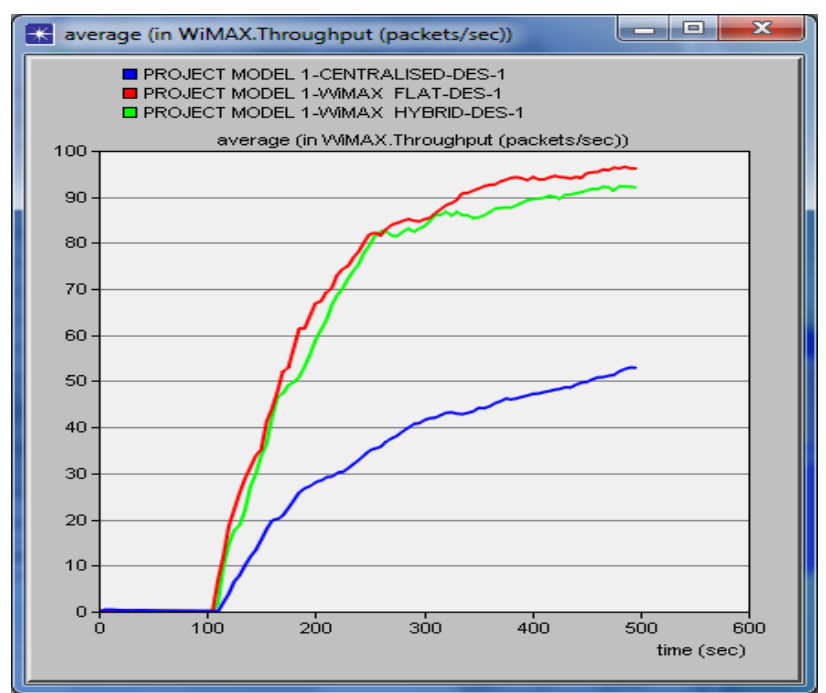

Fig 9: Throughput

\subsection{Uplink Packet Dropped}

Packet drop impacts on the desired quality of the application. Some of the packets drop are as a result of congestion in networks, noise and bit errors in an erroneous network. Packet drop ratio should be minimum, so as to keep the successful delivery of high quality of service. 


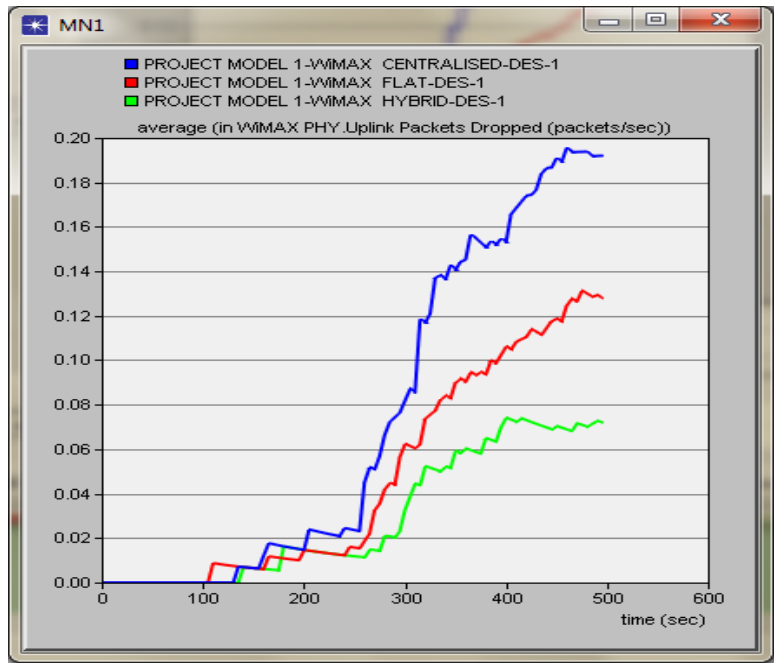

Fig 10: Uplink Packet Dropped

\subsection{Downlink Packet Dropped}

Downlink is the connection between base-stations to mobile nodes, the packet dropped in proposed HCFA architecture is reduced since waiting time of packets are reduced as shown Figure 11.

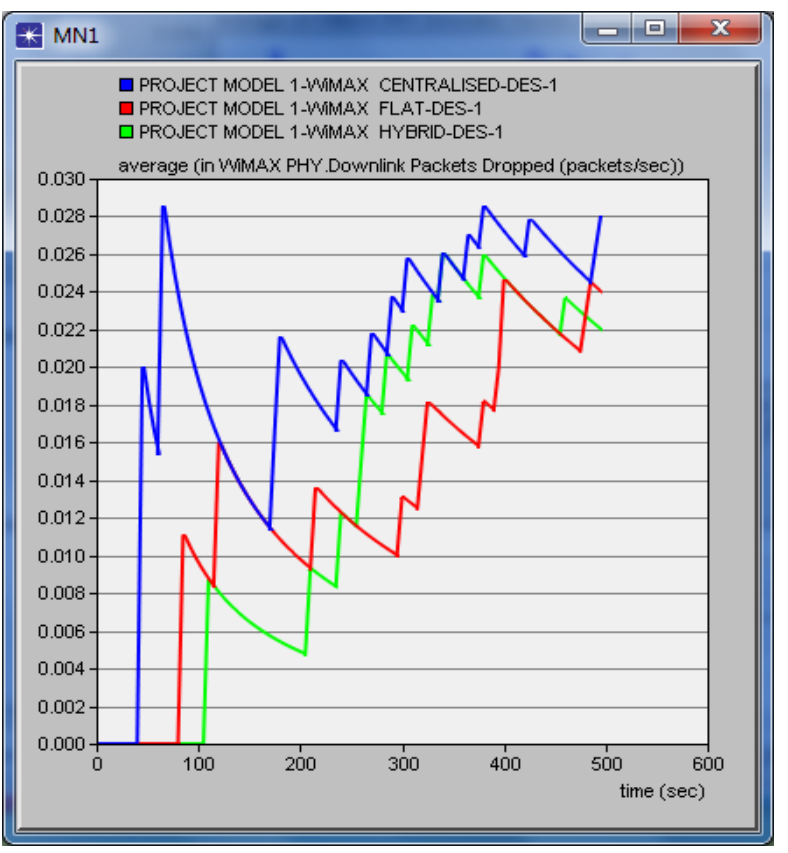

Fig 11: Downlink Packet Dropped

\subsection{Signals to Noise Ratio}

The SNR of uplink shown in Figures 12 Signal to noise ratio is a technical term used to characterize of the signal detected. In centralized architecture, the packet loss is higher and therefore the signal strength is lesser. In flat architecture the packet loss is lesser and therefore the signal strength is higher. Similarly in proposed HCFA architecture the packet loss is reduced and therefore the signal strength is much better than SNR of flat architecture.

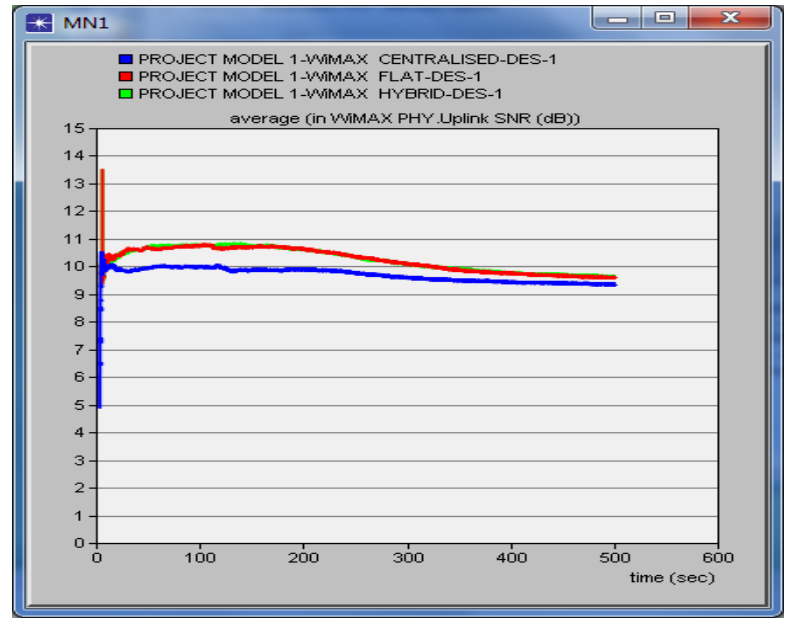

Fig 12: Signal to Noise Ratio

\section{CONCLUSION}

To solve scalability issues and optimize QoS aspects during call establishment and mobility .In this paper the handover performance is analyzed with various WiMAX architectures namely centralized, flat, hybrid coupled flat architecture by using OPNET modeler. Adaptive convergence mechanisms employed for reducing data collision and supporting QoS requirements. ACR control the whole converging mechanism for real time and non real-time services is proposed to enhance the QoS of WiMAX network, it stores information and simultaneously checks for resource availability and handoff control, for every seconds and maintain the database, and adaptively controls the session. Performance result shows that the hybrid coupled flat MWiMAX architecture has improved the QOS in terms of throughput, end to end delay, jitter and packet drop of WiMAX network.

\section{REFERENCES}

[1] IEEE 802.16e-2005: IEEE Standard for Local and Metropolitan Area Networks-Part 16: Air Interface for Fixed and Mobile Broadband Wireless Access Systems

[2] Ed Agis et. Global, Interoperable Broadband Wireless Networks: Extending WiMAX Technology to Mobility. Intel Technology Journal,8(3):173-187, 2004.

[3] IEEE 802.16-2004: IEEE Standard for Local and Metropolitan Area Networks-Part 16: Air Interface for Fixed Broadband Wireless Access Systems.

[4] Sayan Kumar Ray and Harsha irisena."Handover in Mobile WiMAX Networks"The State of Art and Research Issues," IEEE Communications Letters, vol. 12, no. 3, 2010.

[5] R. Q. Huet al. "On the Evolution of Handoff management and Network Architecture in WiMAX." In Proc. IEEE Mobile WiMAX Symposium, pages 144149, Florida, USA, 25-29 March 2007.

[6] Semin Sim and Seung-Jae han, "Seamless IP Mobility Support for Flat Architecture Mobile WiMAX Networks"Tavel, P. 2007 Modeling and Simulation Design. AK Peters Ltd.

[7] K. Daoud, P. Herbelin, K. Guillouard, and N. Crespi, "Performance and Implementation of UFA: a SIP-based Ultra Flat Mobile Network Architecture," in Proceedings of PIMRC 2009, Tokyo, Japan, September 13-16, 2009, pp. 1-6 
[8] Zong-Hua Liu and Jyh-Cheng Chen, "Design and Analysis of the Gateway Relocation and Admissionthe is Control Algorithm in Mobile WiMAX Networks", IEEE Transactions on Mobile Computing, vol. 11, no. 1, pp. 5-18, January 2012.

[9] Ji Hoon Lee, Sangheon Pack, Taekyoung Kwon and Yanghee Choi, "Reducing Handover Delay by Location Management in Mobile WiMAX Multicast and Broadcast Services", IEEE Transactions on Vehicular Technology, vol. 60, no. 2, pp. 605-617, February 2011.

[10] N. Saxena and A. Roy, "Novel framework for proactive handover with seamless multimedia over WLANs," IET Commun., vol. 5, no. 9,pp. 1204-1212, Jun. 2011.

[11] Ray S K, Pawlikowski K, and Sirisena H, A Fast MACLayer Handover for an IEEE 802.16e-Based WMAN,
AccessNets, Chonggang Wang,Ed.: Springer Berlin Heidelberg, 2009, vol. 6, pp. 102-117

[12] Hamdy M, Abdel-Rahman A S, Semary N, Evaluation of Mobile WiMAX IEEE802.16e Handover Load Balancing Trends, The 9thInternational Conference on Informatics and Systems (INFOS2014), December 2014,pp. CNs-25-31

[13] Khatkar A, Singh Y, and Nandal V, A QoS Oriented Analysis of ertPS, rtPS and nrtPS flows in WiMAX, International Journal of Advanced Research in Computer Science and Software Engineering, vol. 3, no. 7, July 2013, pp. 85-90.

[14] D. Lee, D. Won, M. Piran, and D. Suh, "Reducing handover delays for seamless multimedia service in IEEE 802.11 networks," Electron. Lett., vol. 50, no. 15 , pp. 1100-1102, Jul. 2014. 